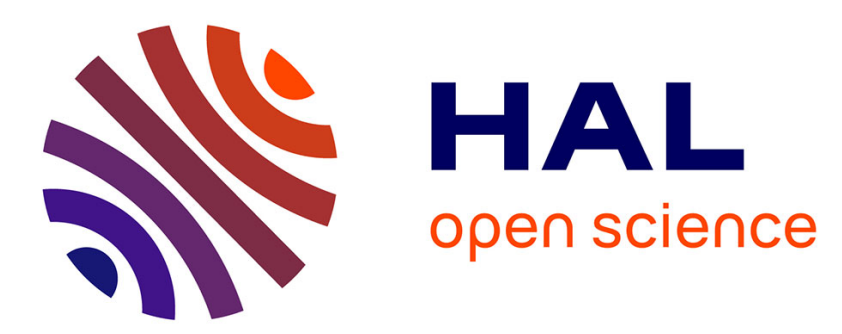

\title{
Conditions d'amorçage de la superposition de puissance entre deux générateurs à plasma
}

\author{
F. Kassabji, Pierre Fauchais
}

\section{To cite this version:}

F. Kassabji, Pierre Fauchais. Conditions d'amorçage de la superposition de puissance entre deux générateurs à plasma. Revue de Physique Appliquée, 1977, 12 (8), pp.1127-1133. 10.1051/rphysap:019770012080112700 . jpa-00244289

\section{HAL Id: jpa-00244289 https://hal.science/jpa-00244289}

Submitted on 1 Jan 1977

HAL is a multi-disciplinary open access archive for the deposit and dissemination of scientific research documents, whether they are published or not. The documents may come from teaching and research institutions in France or abroad, or from public or private research centers.
L'archive ouverte pluridisciplinaire HAL, est destinée au dépôt et à la diffusion de documents scientifiques de niveau recherche, publiés ou non, émanant des établissements d'enseignement et de recherche français ou étrangers, des laboratoires publics ou privés. 


\title{
Classification \\ Physics Abstracts \\ $6.550-6.580-6.700$ \\ CONDITIONS D'AMORÇAGE DE LA SUPERPOSITION DE PUISSANCE ENTRE DEUX GÉNÉRATEURS A PLASMA (*)
}

\author{
F. KASSABJI \\ Laboratoire de Four à Plasma, Centre d'Etudes Nucléaires de Saclay, S. E. C. M. R. G., \\ B. P. n $^{\circ}$ 2, 91190 Gif-sur-Yvette, France \\ et \\ P. FAUCHAIS \\ Laboratoire de Thermodynamique, Université de Limoges, 87100 Limoges, France
}

(Reçu le 30 décembre 1976, accepté le 25 avril 1977)

\begin{abstract}
Résumé. - L'amorçage de la colonne de plasma semble être l'une des difficultés essentielles des différents dispositifs de production d'écoulements permanents de plasma à forte puissance. Nous présentons ici une étude systématique des conditions d'amorçage de la superposition de puissance (courant continu) sur des jets d'argon produits par deux générateurs à arc soufflé et leur évolution en fonction :

- du milieu (air libre ou cavité cylindrique) où se crée la colonne de gaz,

- de la conductibilité électrique du matériau constituant la cavité et de sa géométrie,

- de la puissance électrique, du débit gazeux et de l'écartement des deux générateurs à plasma antagonistes,

— du mode d'écoulement (laminaire, transitoire ou turbulent) qui conditionne la longueur du jet de plasma.
\end{abstract}

\begin{abstract}
The plasma column ignition seems to be one of the main difficulties associated with the different devices for the production of permanent high power plasma flows. The ignition conditions of power superposition on argon jets produced by two plasma generators (D. C.) have been studied. Their evolution depend :

- on the surroundings of the column (free atmosphere or cylindrical cavity)

- on the electrical conductivity of the cavity material, its length and its diameter

- on the electrical power, gas flow rate in the two plasma generators as well as their distances from the cavity

- on the flow type (laminar, transitory and turbulent) which governs the jet length.
\end{abstract}

1. Introduction. - Les diverses applications utilisant comme source de chaleur des générateurs à plasma à arc exigent pour passer à l'échelle industrielle une plus grande puissance dissipée, une température moyenne plus élevée et un flux de chaleur plus important.

Les pertes par le refroidissement des anodes étant en première approximation, pour un débit et un gaz donné, proportionnelles à l'intensité et à la tension à

(*) Ce travail effectué sous convention d'étude C. E. A./E. D. F. (S. A. V. 1934) et contrat C. E. A./Université de Limoges $n^{\circ} 5323$ se rapporte à la thèse de Docteur-Ingénieur de F. Kassabji. Communication présentée au Congrès National de Physique des Plasmas, Paris, 6-10 décembre 1976. une puissance $n$ ( $n$ compris entre 0,1 et 0,3 ), il est préférable pour augmenter la densité de puissance fournie au gaz, d'augmenter la tension de préférence à l'intensité [1]. Pour cela on peut allonger le trajet de l'arc dans le gaz plasmagène et établir des colonnes de plasma avec un champ de 3 à $20 \mathrm{~V} / \mathrm{cm}$ (selon la nature du gaz) pour des intensités de 300 à $3000 \mathrm{~A}$.

Les principaux dispositifs pour obtenir des écoulements permanents de plasma de forte puissance sont les suivants :

a) les arcs transférés où l'une des deux électrodes (la tuyère) ne sert qu'à la stabilisation de l'arc, transféré à une électrode externe [2], [3] ;

b) les arcs superposés avec superposition de puissance en courant alternatif ou continu sur le conduc- 
teur gazeux formé par deux [4], [5] et [6] ou plusieurs jets de plasma concourants [7], [8] et [9] ;

c) les arcs superpo-transférés : association des deux systèmes précédents [10].

Nous proposons ici d'étudier les différents paramètres influençant la création et le maintien d'une colonne de plasma établie à l'air libre ou confinée dans une cavité cylindrique obtenue en superposant une puissance continue sur deux jets de plasma produits par des générateurs à arc fonctionnant eux-mêmes en courant continu. Une telle configuration est particulièrement intéressante pour le développement des fours rotatifs à plasma tant verticaux [10] représentés sur la figure 1 qu'horizontaux [6] représentés sur la figure 2. En effet, actuellement la puissance de ces dispo-

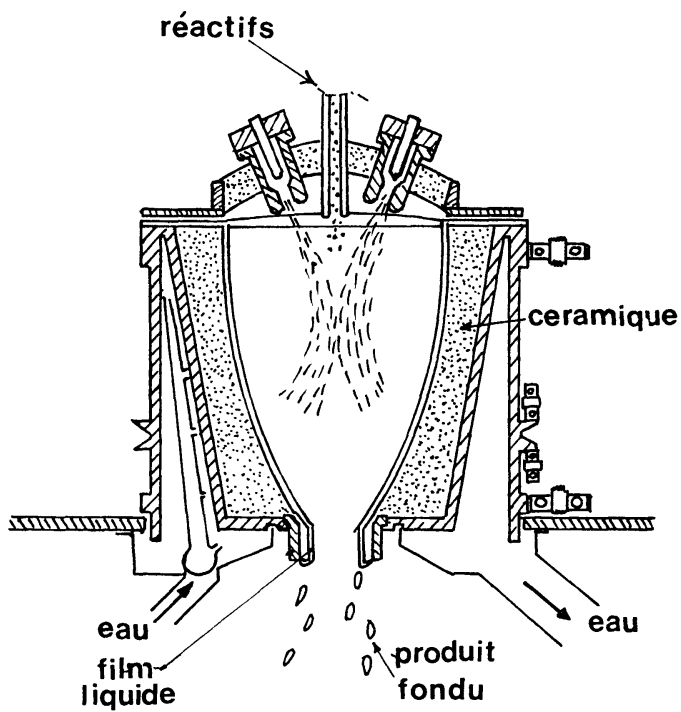

Fig. 1. - Four à plasma centrifuge vertical [10]. [Vertical plasma furnace [10].]

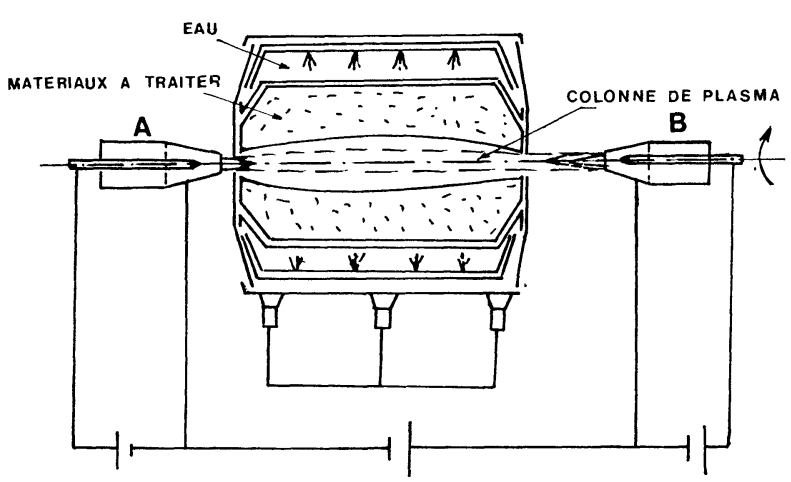

FIG. 2. - Four à plasma horizontal [6].

[Horizontal plasma furnace [6].]

sitifs est limitée à $100 \mathrm{~kW}$ et pour l'augmenter il convient en particulier d'accroître la longueur du jet de plasma produit ce qui corrélativement rend très difficile son amorçage.
2. Définition des paramètres de fonctionnement. Nous nous proposons donc d'étudier notamment pour le dispositif de superposition (schématisé sur la Fig. 3) les conditions d'obtention d'une colonne de plasma à forte puissance. Nous avons toutefois limité notre étude à des jets de plasma d'azote ou d'argon qui sont les plus couramment utilisés dans ce type de four.

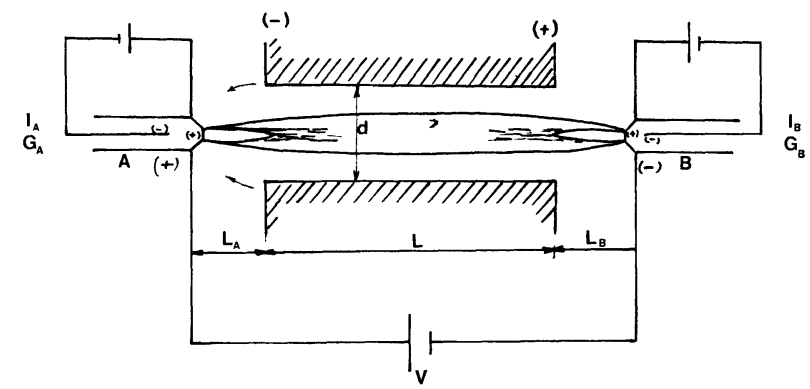

FIG. 3. - Superposition de courant continu entre deux générateurs à plasma.

[Superposition of direct current between two plasma generators.]

Les jets produits par deux générateurs à plasma en courant continu servent de support à une superposition de puissance en courant continu formant ainsi une colonne de plasma qui joue le rôle d'une résistance gazeuse chauffant par rayonnement l'espace et la matière environnante.

La création de cette colonne passe par les trois phases suivantes :

1. phase de préchauffage (constituant une phase de perte) ;

2. phase d'amorçage ;

3. phase de maintenance ou fonctionnement.

Nous schématisons ces trois phases en considérant la variation de la puissance dissipée dans cette colonne en fonction du temps sur la figure 4.

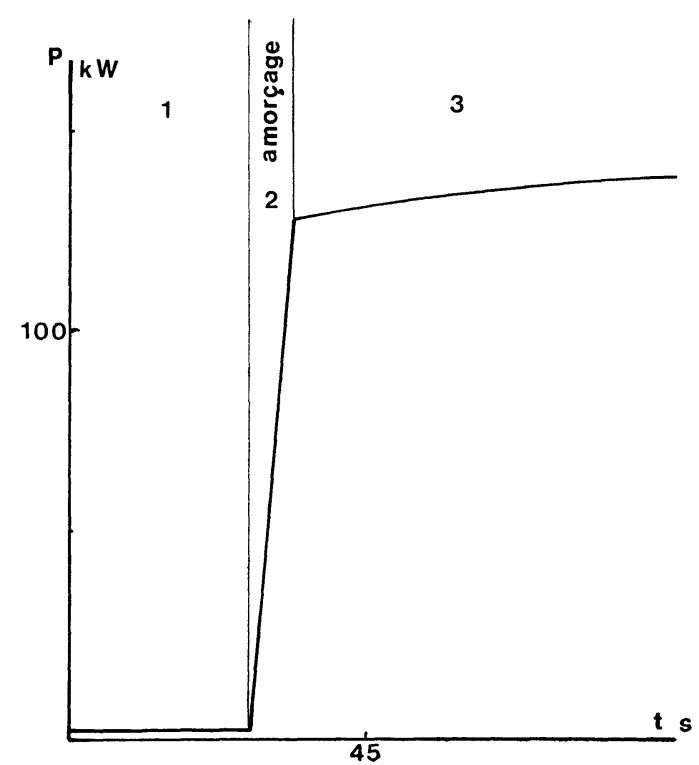

Fig. 4. - Phases de création de la colonne de plasma. [The phases of creation of a plasma column.] 
Afin d'améliorer le fonctionnement du four à plasma à axe horizontal dont nous disposons à Saclay, nous avons essentiellement étudié ce dispositif dans le cas des deux générateurs placés l'un en face de l'autre (à $\left.180^{\circ}\right)$ (Fig. 3) en superposant la puissance soit à l'air libre soit dans un espace donné.

La création de la colonne de plasma entre les anodes des deux générateurs à plasma, les jets produits servant d'électrodes gazeuses, est influencée par différents paramètres permettant de favoriser et d'optimiser le processus. Nous pouvons en donner une énumération non exhaustive : la tension à vide de la source de courant alimentant le circuit de superposition, la géométrie des générateurs à plasma, leur puissance, les débits et la nature du gaz, la longueur des jets produits le milieu de la formation de la colonne, la nature et la géométrie de ce milieu, etc...

Pour la simplification de l'étude, et étant donné l'influence indépendante mais complémentaire de plusieurs de ces paramètres, nous les avons classés en trois catégories :

1. Alimentation électrique de la colonne:

Le plus important étant la tension à vide nécessaire à l'amorçage.

\section{Générateurs à plasma :}

a) la longueur des jets produits ou la forme de l'écoulement produit,

b) la nature du gaz dans chaque générateur,

c) le débit $G$ du gaz dans chaque générateur,

d) l'intensité électrique et la puissance dans chaque générateur.

3. Milieu de fonctionnement :

a) nature de ce milieu,

- air ou mélange gazeux,

- corps conducteurs ou non conducteurs électriques servant au confinement;

b) géométrie de ce milieu.

Cavités cylindriques rotatives ou non, ayant des diamètres et des longueurs différents.

3. Alimentation électrique de la superposition. La création de la colonne de plasma est liée essentiellement à la tension établie entre les deux jets de plasma. La tension de claquage dépend principalement de la distance entre les deux générateurs et plus précisément entre les deux jets produits jouant le rôle d'électrodes gazeuses, de la forme des électrodes et de leur polarité, et de la nature et du débit du gaz.

A l'air libre, il a pu être montré [11] qu'en fonction de la distance les valeurs de tension de claquage $U$ suivent une loi simple :

$$
U_{50}=\frac{3400}{1+8 / d} \text { en } \mathrm{kV}
$$

$d$ étant la distance entre les deux pôles en $\mathrm{m}$.
Toutefois, avec les plasmas ces tensions très élevées de claquage peuvent être évitées en créant entre les électrodes un milieu gazeux conducteur électrique. On doit cependant souligner que les essais d'amorçage à l'air libre entre deux jets de plasma concourants ont montré que la valeur minimale de la tension à vide de la source de superposition doit être au moins de l'ordre de 3 fois la tension de fonctionnement du système. Cette tension de fonctionnement est naturellement liée à la distance séparant les deux générateurs et elle augmente légèrement avec l'intensité suivant une caractéristique définie de l'arc ; la longueur maximale de la colonne de plasma obtenue est d'autant plus faible que l'intensité est basse. La figure 5 représente la variation de la tension en fonction de $L$ pour différentes intensités dans le cas du fonctionnemenı à l'air libre de jets de plasma d'argon concourants. Une loi similaire est obtenue avec des jets de plasma d'azote.

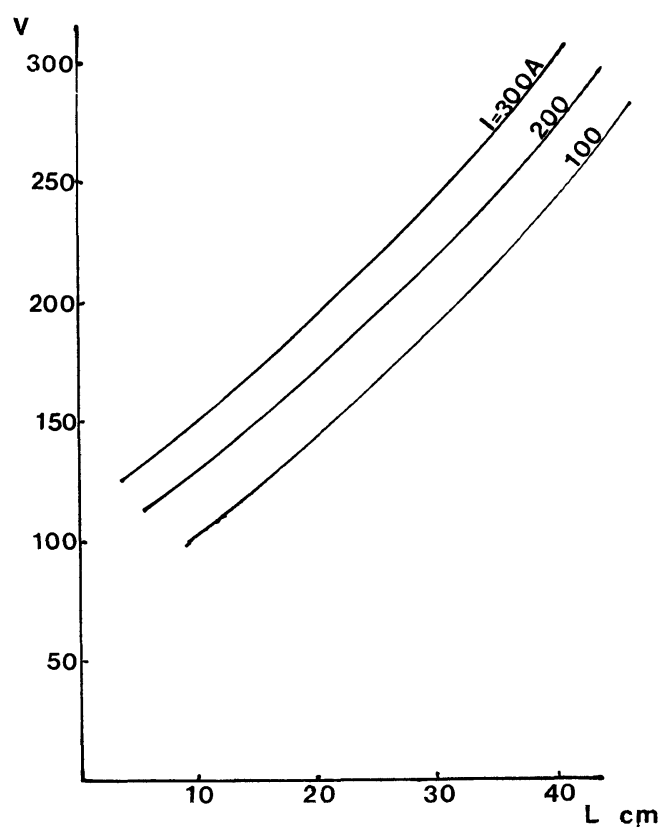

Fig. 5. - Variation de la tension en fonction de la distance pour différentes intensités dans le cas de fonctionnement à l'air libre, pour des débits d'argon dans les générateurs $G_{\mathrm{A}}=G_{\mathrm{B}}=15 \mathrm{l} / \mathrm{min}$.

[Voltage variation depending on the distance between the two generators working in air and for different intensities, for argon flows in the generators $G_{\mathrm{A}}=G_{\mathrm{B}}=15 \mathrm{l} / \mathrm{min}$.]

Dans le four à axe horizontal, le four est chargé au départ avec les matériaux à traiter (sous forme divisée) et seul un espace cylindrique est laissé libre le long de l'axe pour permettre le passage du jet de plasma et $y$ obtenir une densité de puissance importante. C'est pour cela que nous avons étudié l'amorçage à travers un tube de longueur et de diamètre donnés tant conducteur électrique (graphite) qu'isolant (alumine). Ceci exige une tension plus faible, car les jets créés sont constrictés par leur passage dans ce tube ce qui contribue à leur stabilité et leur allongement, et l'espace 
emprisonné est limité en volume ce qui facilite le chauffage du gaz.

La croissance du diamètre intérieur du tube augmente la valeur de la tension à vide exigée pour cet amorçage. En effet, un volume plus petit de gaz emprisonné, bien que se renouvelant par partie par les orifices, atteint plus rapidement une température permettant une ionisation suffisante pour l'amorçage de la colonne de plasma.

La figure 6 présente pour des conditions données du fonctionnement des générateurs et des dispositions

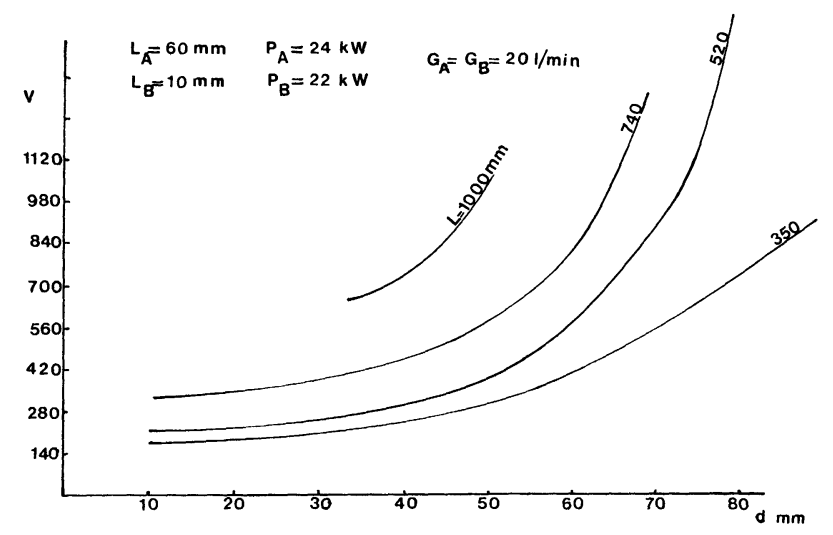

Fig. 6. - Influence de l'augmentation du diamètre sur la tension minimale de l'amorçage.

[The influence of increase of the diameter on the minimum voltage of the column ignition.]

géométriques définies, l'influence de l'augmentation du diamètre sur la tension minimale exigée pour amorcer la colonne de plasma. On tend rapidement vers une limite au-delà de laquelle la valeur de la tension à vide devient très élevée.

4. Les générateurs à plasma. - Les deux générateurs à plasma produisant les jets servant de support à la superposition interviennent dans le système de l'amorçage au niveau de leur puissance, de leur géométrie, du débit et de la nature du gaz plasmagène, ainsi que de leurs distances respectives aux extrémités de la cavité intermédiaire (pour une longueur et un diamètre fixé de celle-ci). Tous les résultats donnés dans ce qui suit ont été obtenus avec des plasmas d'argon, mais ils sont similaires avec l'azote.

4.1 INFLUENCE DES DÉBITS $\boldsymbol{G}$. - Les débits d'argon dans chacun des deux générateurs peuvent être modifiés en cours de fonctionnement ce qui influe sur la valeur de la tension de superposition aux bornes de la colonne de plasma formée.

Pour une intensité de superposition constante, d'une part, la tension augmente avec le débit total des deux générateurs $\left(G_{\mathrm{A}}+G_{\mathbf{B}}\right)$ et d'autre part, cette augmentation est d'autant plus sensible et atteint son maximum pour des débits égaux dans les deux générateurs $\left(G_{\mathrm{A}}=G_{\mathrm{B}}\right)$ (Fig. 7).

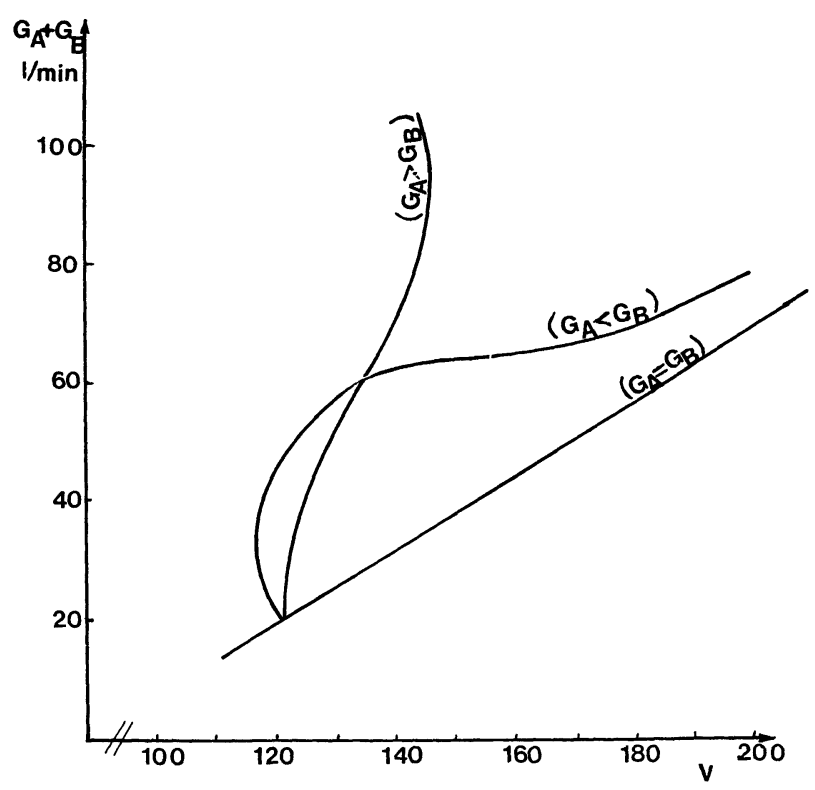

Fig. 7. - Influence du total des débits d'argon sur la valeur de la tension de fonctionnement, pour une intensité de superposition constante $I_{\mathrm{s}}=250 \mathrm{~A}$.

[The influence of the total argon flow on the value of the work tension for a constant superposition intensity $I_{\mathrm{s}}=250 \mathrm{~A}$.]

4.2 INFLUENCE DES PUISSANCES DANS CHAQUE GÉNÉRATEUR. - La détermination des puissances utiles pour l'amorçage permet de définir le type de générateur à plasma.

Pour un gaz donné et un débit égal dans chaque générateur, ces puissances (ou ces intensités dans les conditions définies) sont liées aux distances des générateurs aux extrémités du tube. Nous représentons sur la figure $8\left(I_{\mathrm{A}}=f\left(I_{\mathrm{B}}\right)\right.$ en fonction de $l_{\mathrm{A}}$ et $\left.l_{\mathrm{B}}\right)$ les courbes séparant la zone de l'amorçage immédiat de celle où l'amorçage est très difficile sinon impossible.

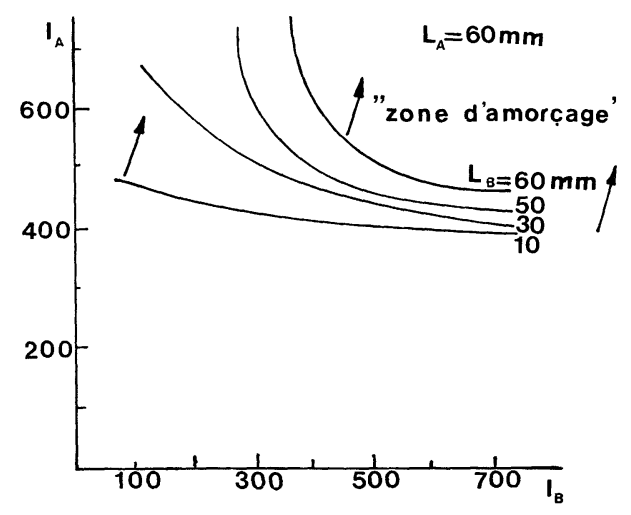

Fig. 8. - Influence des puissances dans les générateurs pour différentes distances $\left(l_{\mathrm{B}}\right)$ séparant le générateur à plasma $\mathrm{B}$ de la cavité cylindrique et pour la distance $l_{\mathrm{A}}=60 \mathrm{~mm}$ sur l'amorçage de la colonne dans le cas d'un débit d'argon dans les deux générateurs : $G_{\mathrm{A}}=G_{\mathrm{B}}=20 \mathrm{l} / \mathrm{min}$.

[The influence of the generator powers on the column ignitions for different distances $l_{\mathrm{B}}$ between the plasma generator $\mathrm{B}$ of the cylindrical cavity and for a distance $l_{\mathrm{A}}=60 \mathrm{~mm}$, for the case of argon flow in the two generators: $G_{\mathrm{A}}=G_{\mathrm{B}}=20 \mathrm{l} / \mathrm{min}$.] 
Pour une valeur donnée de $l_{\mathrm{A}}$ ou de $l_{\mathrm{B}}$, l'amorçage est très facile avec des valeurs de $I_{\mathrm{A}}$ et $I_{\mathrm{B}}$ se situant dans la zone au-delà de la courbe correspondante.

4.3 INFLUENCE DE LA FORME DE L'ÉCOULEMENT. Il est important de signaler qu'il est très difficile d'obtenir une colonne de superposition entre deux jets (toute réserve faite pour des tensions à vide excessivement élevées) s'ils ne se touchent au moins par leur panache. Il est donc nécessaire de pouvoir disposer de deux jets les plus longs possibles pour amorcer la superposition de la puissance à de grandes distances donc d'allonger le trajet du gaz plasmagène et d'augmenter par la suite la tension et la puissance utile.

Trois formes d'écoulement sont représentatives du jet produit, les nombres de Reynolds les caractérisant sont les suivants :

$R e<110$ à 265 l'écoulement est laminaire 110 à $265<R e<300$ à 845 l'écoulement est transitoire $R e>300$ à 845 l'écoulement est turbulent.

Notons que la détermination de la forme de cet écoulement repose sur des mesures expérimentales, soit visuelles : les jets lumineux les plus longs (jusqu'à $60 \mathrm{~cm}$ ) et dont les températures restent inférieures à $8000 \mathrm{~K}$ sont obtenus pour des écoulements laminaires, les jets turbulents étant moins longs (quelques centimètres) et très brillants $(T>10000 \mathrm{~K})$ [4], [12] ; soit soniques : le niveau du bruit de fond enregistré pour un écoulement laminaire (quelques dizaines de décibels), s'amplifie pour atteindre des valeurs supérieures à $100 \mathrm{~dB}$ avec un écoulement turbulent [4], [13], [14]. Le manque de précision de ces mesures pour caractériser l'écoulement ainsi que les différents gaz utilisés expliquent la marge indiquée pour les valeurs du nombre de Reynolds se rapportant à chaque forme d'écoulement.

En nous appuyant sur les valeurs des différentes propriétés des gaz ou des mélanges gazeux [15], nous avons pu déterminer la relation entre le diamètre de la tuyère et le débit volumique du gaz permettant l'obtention d'un écoulement laminaire ; cette relation est de la forme

$$
G_{\mathrm{gaz}}<a d
$$

$G_{\text {gaz }}$ étant le débit volumique du gaz ou du mélange gazeux injecté en $1 / \mathrm{min}$.

$d$ le diamètre de la tuyère anode en $\mathrm{mm}$.

a une constante variant avec la nature du gaz ou du mélange.

Elle est de 2,31 pour l'azote pur, 1,92 pour l'argon pur,

12,25 pour l'hydrogène pur.

La géométrie du générateur à plasma joue aussi un rôle important dans la production d'une forme convenable du jet, un très bon centrage cathode-anode étant capital. Le mode d'injection axiale du gaz permet d'éviter des turbulences gênantes pour l'écoulement laminaire souhaité. La stabilité du jet dans ce cas est assurée par la couche gazeuse froide protectrice se formant le long des électrodes. L'éventuelle usure plus rapide de l'anode peut être évitée par un refroidissement très efficace [4], [13].

4.4 INFLUENCE DES DISTANCES GÉNÉRATEUR-TUBES. - Des essais systématiques d'amorçage de la superposition dans des cavités cylindriques ont permis de déterminer l'influence des distances $\left(l_{\mathrm{A}}\right.$ et $\left.l_{\mathrm{B}}\right)$ séparant les générateurs des extrémités du tube (Fig. 1). Ces distances sont déterminantes pour la possibilité de l'amorçage rendu très difficile au-dessus d'une valeur maximale de $l_{\mathrm{A}}$ ou de $l_{\mathrm{B}}$.

La figure 9 représente la relation existant entre les deux distances $l_{\mathbf{A}}$ et $l_{\mathbf{B}}$ en délimitant en particulier

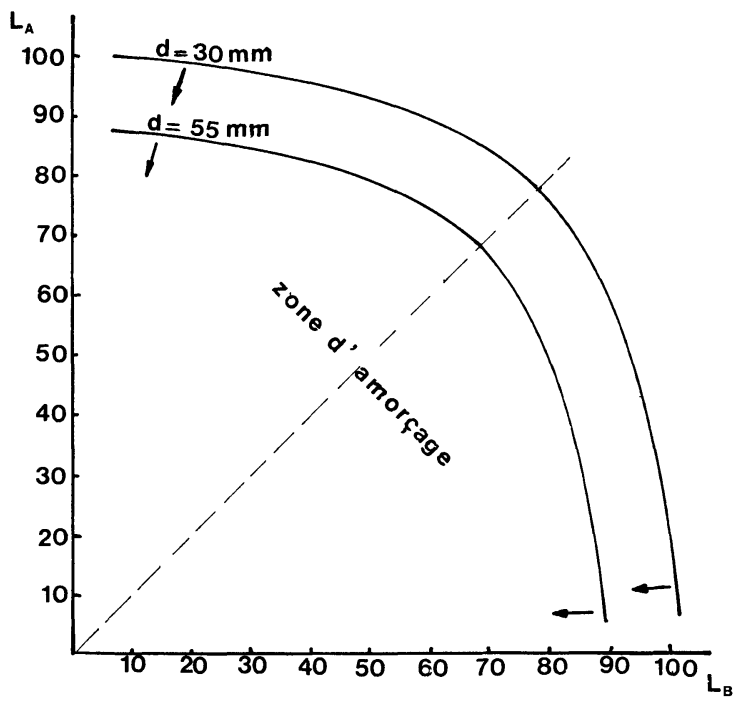

FIG. 9. - Relation entre les deux distances respectives des deux générateurs à des cavités cylindriques ayant différents diamètres, les puissances dans les générateurs étant $P_{\mathrm{A}}=22 \mathrm{~kW}$ et $P_{\mathrm{B}}=21 \mathrm{~kW}$ avec des débits $G_{\mathrm{A}}=G_{\mathrm{B}}=201 / \mathrm{min}$.

[The relation of the two respective distances between the two generators and the cylindrical cavities of different diameters, the powers in the generators are $P_{\mathrm{A}}=22 \mathrm{~kW}$ and $P_{\mathrm{B}}=21 \mathrm{~kW}$ with argon flow $G_{\mathrm{A}}=G_{\mathrm{B}}=20 \mathrm{l} / \mathrm{min}$.]

deux zones d'accrochage : facile et rapide d'une part et difficile et long d'autre part. Ces deux zones sont séparées par une courbe symétrique par rapport à la diagonale délimitant avec les axes $x$ et $y$ la zone d'accrochage optimale.

Pour des tubes ayant une longueur ne dépassant pas $75 \mathrm{~cm}$ dans le cas des matériaux non conducteurs à froid $\left(\mathrm{Al}_{2} \mathrm{O}_{3}\right.$ ou $\left.\mathrm{MgO}\right)$ et $100 \mathrm{~cm}$ dans le cas des matériaux conducteurs (graphite) et pour des diamètres variant entre 30 et $80 \mathrm{~mm}$, la formule empirique déterminée est

$$
l_{\mathrm{A}}=\frac{4}{l_{\mathrm{B}}-9}+9
$$

où $l_{\mathrm{A}}$ et $l_{\mathrm{B}}$ sont les distances des deux générateurs $\mathrm{A}$ et $\mathrm{B}$ aux extrémités du tube (en $\mathrm{cm})$. 
Dans le cas de corps conducteurs en graphite nous remarquons qu'à l'amorçage de la colonne de plasma entre les deux jets, de petits arcs secondaires ou parasites se forment du côté du générateur à plasma chargé positivement. La création de ces arcs est due :

4.4.1 à l'émission thermoélectronique et la pulvérisation cathodique : le potentiel d'extraction du matériau augmentant avec la température facilite l'arrachement des particules à l'extrémité chaude du tube chargé négativement (en face du pôle positif de l'amorçage sur le générateur). Ces particules attirées par la charge positive peuvent ou bien attaquer le cuivre refroidi de l'anode et la détruire (usure rapide) ou bien pour une distance suffisamment grande permettre la dispersion de l'énergie contenue dans ces particules et se confondre avec la colonne formée ;

4.4.2 aux effets de pointe : au phénomène précédent peut s'ajouter pour des tubes encore non usés les effets de pointe. Des particules sont arrachées du bout du tube pointu par la concentration d'énergie, et sont attirées par la charge positive, elles agissent comme précédemment en attaquant le cuivre ou en se confondant avec la colonne de plasma suivant la distance.

Il est donc nécessaire de ne pas descendre en dessous d'une distance minimale pour éviter une usure rapide et destructive de l'anode du générateur à plasma du côté positif de la superposition. Tandis que du côté négatif le phénomène de formation des arcs parasites n'est pas remarqué car le cuivre refroidi par eau ne permet aucune thermoémission.

5. Milieu de fonctionnement. - 5.1 A L'AIR LIBRE. - Les jets de plasma produits par les deux générateurs placés à différentes inclinaisons doivent concourir en un même point. L'amorçage de la colonne est étroitement lié à la longueur des jets et plus précisément à leur rencontre au moins par leur panache, une boule chaude se formant alors dans la zone de rencontre [4], [10]. A titre d'exemple, nous donnons quelques caractéristiques de fonctionnement du dispositif à l'air libre dans le cas des deux générateurs placés l'un en face de l'autre (à $180^{\circ}$ ).

Générateur à plasma $\mathrm{A}$ :

$$
\begin{gathered}
\text { débit d'argon } 12 \mathrm{l} / \mathrm{min}, \quad V=22 \mathrm{~V}, \\
I=600 \mathrm{~A}, \quad P=13,2 \mathrm{~kW} ;
\end{gathered}
$$

Générateur à plasma $\mathrm{B}$ :

$$
\begin{gathered}
\text { débit d'azote } 12 \mathrm{l} / \mathrm{min}, V=70 \mathrm{~V}, \\
I=300 \mathrm{~A}, \quad P=21 \mathrm{~kW} ;
\end{gathered}
$$

Superposition :

$$
V=300 \mathrm{~V}, \quad I=300 \mathrm{~A}, \quad P=90 \mathrm{~kW} ;
$$

distance entre les deux générateurs $=42 \mathrm{~cm}$.

Les pertes de chaleur étant essentiellement localisées dans les anodes refroidies par eau des deux générateurs, le rendement dans la colonne de plasma atteint 80 à $85 \%$.
5.2 CORPS CONDUCTEURS ET NON CONDUCTEURS CONSTITUANT L'ENCEINTE. - Les caractéristiques de la colonne de plasma guidée dans une enceinte sont différentes de celles à l'air libre. Cette enceinte permet d'une part, le confinement des jets produits et les rallonge considérablement et d'autre part, le chauffage du gaz emprisonné à l'intérieur et par suite son ionisation facilitant ainsi l'amorçage.

Une dérivation du courant peut se produire dans le cas des corps conducteurs (graphite par exemple) et des arcs parasites peuvent se former en établissant le circuit du courant dans la matière entre les extrémités du tube et les jets de plasma. Ces arcs parasites sont établis surtout pour des puissances relativement faibles et durent quelques secondes jusqu'à l'ionisation du gaz dans la cavité et donc la diminution de sa résistance et l'établissement de la colonne de plasma à des puissances importantes.

Pour les corps non conducteurs, on ne remarque pas d'arcs parasites mais l'amorçage de la colonne est plus long. Des particules du matériau $\left(\mathrm{MgO}, \mathrm{Al}_{2} \mathrm{O}_{3}\right.$ ou mélanges) sont projetées à l'extérieur attaquant quelquefois les anodes des générateurs.

6. Paramètres principaux et conclusion. - Une méthode statistique de carré latin orthogonal permet-

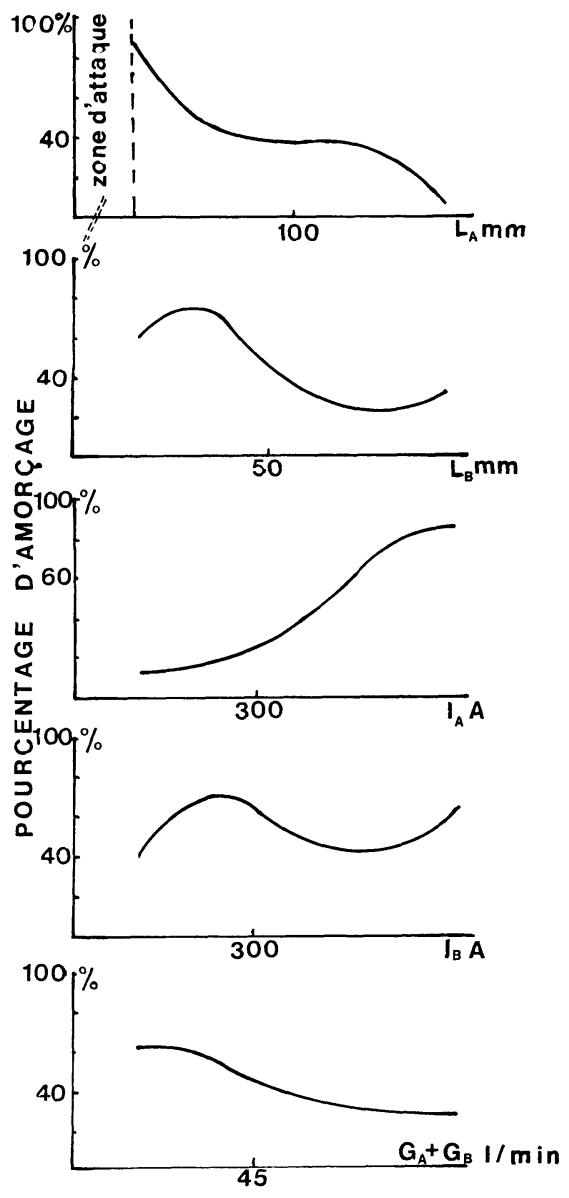

FIG. 10. - Détermination des paramètres principaux influençant l'amorçage de la colonne de plasma.

[The determination of the principal parameters influencing the plasma column ignition.] 
tant la comparaison simultanée de plusieurs paramètres indépendamment variables les uns des autres et agissant sur une même opération, a été employée. On a cherché parmi tous les paramètres influençant une opération d'amorçage de la colonne de plasma ceux qui jouent un rôle prioritaire ou qui sont les plus importants dans la création de cette colonne. Cinq paramètres variant simultanément ont été comparés : les deux distances $l_{\mathrm{A}}$ et $l_{\mathrm{B}}$ des générateurs aux extrémités du tube, les deux intensités ou puissances dans les deux générateurs et la somme des débits de gaz injectés (en se plaçant dans le cas optimal des deux débits égaux dans les deux générateurs). La figure 10 représente l'influence des paramètres précédents sur l'amorçage de la colonne de plasma.

Nous remarquons l'importance des distances $l_{\mathbf{A}}$ et $l_{\mathrm{B}}$ sur l'amorçage et l'existence de la zone d'attaque ; les intensités (puissances) des générateurs sont liées à ces distances et leur augmentation améliore le fonctionnement jusqu'à un maximum au-dessus duquel l'influence de la puissance est moindre ; quant à la somme des débits, son influence est importante pour les faibles débits puis, après stabilisation, négligeable pour les forts débits.

En conclusion ce travail est le début d'une étude de la superposition de puissance entre deux générateurs à plasma en courant continu afin de réaliser une colonne de plasma à forte densité d'énergie telle qu'on en utilise dans les fours à plasma rotatifs tant à axe vertical qu'horizontal. Les différents paramètres étudiés nous ont permis de déterminer les conditions d'amorçage optimal de la colonne de plasma :

- tension à vide minimale (deux à trois fois la tension de la colonne);

- distance minimale des générateurs à plasma aux cavités cylindriques permettant de limiter considérablement l'usure des anodes lors du transport ;

- puissance minimale nécessaire dans chacun des deux générateurs pour maintenir la superposition.

Ces résultats seront prochainement généralisés avec divers dispositifs d'écoulement de plasma de forte puissance.

\section{Bibliographie}

[1] Fauchais, P., Les Hautes Températures et leurs utilisations en physique et en chimie (Masson et Cie), Tome 1, p. 41.

[2] Gross, B., Grycz, B., Miklossy, K., Plasma Technology (ICIFFE Books LTD : London) 1968.

[3] BRowning, J., Dynamics of conducting gases proceedings of the Third biennal gas dynamics symposium (Northwestern University Press) 1960 p. 126.

[4] KassabJi, F., Thèse Doctorat $3^{\mathrm{e}}$ cycle, Limoges 1975.

[5] Aubreton, J., Dallaire, S., Yerouchalmi, D., Colloque du Comité Français d'Electrothermie, Versailles 1974.

[6] Blatman, H., Delmas, R., FoeX, M., Yerouchalmi, D. Brevet 95689 C. E. A. France.

[7] Schoumaker, H., Communication au C. B. E. E. (1971).
[8] Bonet, C., High Temp. High Pressures 3 (1971) 333.

[9] Bonet, C., Lamos, J., Foex, M., Entropie 34-35 (1970) 36.

[10] HowIe, F. H., SAYce, I. G., Rev. Int. Hautes Temp. Réfract. 11 (1974) 169.

[11] Boillot, A., AliI, Rev. Gén. Electr. 83 (1974) 761.

[12] INCRoperA, F. P., Flow Transition Phenomena in a Subsonic plasma jet (T. R.-SU 247-9), August 1965.

[13] Fauchais, P., Thèse Doctorat ès sciences, Poitiers 1968.

[14] InCROPERA, F., LePPERT, G., Flow Transition Phenomena in a Subsonic Plasma jet (AIAA Journal), vol. 4, $\mathrm{n}^{\circ}$, June 1966.

[15] Gorse, C., Thèse Doctorat $3^{e}$ cycle, Limoges 1975. 